\title{
Estudo das Trincas Retas com o Método dos Elementos de Contorno, a Função de Green Numérica e a Técnica da Dupla Reciprocidade
}

\author{
C.A.R. VERA-TUDELA e M.F. CORSI
}

Recebido em 5 de dezembro de 2017 / Aceito em 27 de outubro de 2018

\begin{abstract}
RESUMO. Nos últimos anos, o Método dos Elementos de Contorno (MEC) tem sido aplicado com sucesso a problemas da mecânica da fratura linear elástica (MFLE) envolvendo os casos estático e dinâmico. Para resolver problemas com ações de domínio (por exemplo: forças gravitacionais, problemas transientes com velocidades e acelerações, etc.) via MEC, Nardini e Brebbia apresentaram em 1982 a técnica da Dupla Reciprocidade (DR), inicialmente com a intenção de resolver problemas transientes usando soluções fundamentais estáticas, mas que se revelou bastante adequado e eficaz na solução de problemas com ações de domínio. Com base no acima exposto, este trabalho apresenta estudos complementares iniciados por Vera-Tudela em 2003, utilizando a técnica da Função de Green numérica (FGN), junto com a técnica da Dupla Reciprocidade. O desenvolvimento destas três formulações para a analise de problemas da mecânica da fratura sob o efeito de cargas de domínio constituem um avanço na abordagem dos problemas através do MEC. Embora o uso da técnica da Dupla Reciprocidade em problemas da mecânica da fratura não seja recente, sua implementação conjunta com a Função de Green numérica representa uma novidade na pesquisa e com a vantagem de não precisar de se efetuar a discretização das superfícies das trincas pois as integrações são feitas somente sobre o contorno do problema. Dois exemplos são apresentados e os resultados quando comparados com a bibliografia de referência e com a teoria mostram e eficiência da formulação.
\end{abstract}

Palavras-chave: Método dos Elementos de Contorno, Função de Green Numérica, Dupla Reciprocidade.

\section{INTRODUÇÃO}

As trincas ocupam um lugar muito importante nas causas de falha dos componentes de máquinas ou na construção civil. Praticamente todo elemento fabricado apresenta alguma trinca que pode ser causada pela movimentação de materiais na confecção, por algum tratamento térmico ou ainda como conseqüência da ocorrência de vibração na área. Na verdade, as causas para o aparecimento de trincas são numerosas e dependem de muitos fatores como o próprio material, condições de trabalho do elemento ou até condições externas. As trincas são a principal causa de

\footnotetext{
*Autor correspondente: C. A. R. Vera-Tudela - E-mail: candres@ufrrj.br - https://orcid.org/ 0000-0001-5855-8611

Universidade Federal Rural do Rio de Janeiro, Departamento de Matemática, PPG em Modelagem Matemática e Computacional, Caixa Postal 74517, 23897-970, Seropédica, RJ, Brasil. E-mails: candres@ufrrj.br, ofmarlon@yahoo.com.br
} 
diminuição da resistência do material, o que pode levar ao colapso do elemento. Desta forma, a necessidade de estudar trincas foi ganhando importância devido ao seu envolvimento com fatores econômicos, da própria vida útil do equipamento e da segurança das pessoas.

Pesquisadores vêm trabalhando neste sentido, desenvolvendo diversas técnicas de forma a prever o comportamento de trincas e, em consequiência, a vida útil de componentes. Diversas propostas vêm sendo apresentadas com êxito e o número de publicações científicas demonstra a importância que a Mecânica da Fratura Linear Elástica (MFLE) tem na engenharia.

Dentre todo este universo destaca-se como ferramenta o Método dos Elementos de Contorno (MEC) que vem sendo aplicado com êxito a diversas propostas de resolução de problemas de engenharia, seja no caso estático ou no dinâmico, uma vez que os resultados obtidos apresentam alta precisão, com a vantagem adicional da discretização somente do contorno do problema. No campo da mecânica da fratura os registros mostram que foi Cruse, 1972, o primeiro a se aprofundar no estudo do MEC como formulação aplicada à MFLE. Na atualidade, pode-se considerar que três alternativas do MEC são predominantes no estudo das trincas. A primeira é uma extensão do método da sub-região [3] onde divide-se o corpo trincado em duas regiões e cada face da trinca pertence a um domínio. Na superfície de interface entre as regiões, a compatibilidade de deslocamentos e o equilíbrio de tensões são impostos. A segunda é a formulação mista [8] [12] baseada na equação dos deslocamentos e de suas derivadas na direção normal. Assim, cada equação integral, a clássica e a hipersingular, é aplicada a uma superfície diferente da trinca evitando-se o uso de subregiões que introduzem um contorno artificial no problema. A terceira utiliza a Função de Green correspondente ao problema [9] que permite a representação exata de trincas pela equação integral de contorno, sem a necessidade de se efetuar a discretização das mesmas: as integrações são feitas somente sobre o contorno externo do problema e a formulação apresenta uma excelente precisão.

Assim, o campo da Mecânica da Fratura caracteriza-se por ser interdisciplinar e oferece uma fonte importante para novos descobrimentos, e novos resultados dão a oportunidade de servir em aplicações imediatas.

O estudo da Mecânica da Fratura está relacionado com a descrição quantitativa de um estado mecânico num corpo elástico contendo uma ou mais trincas. O processo de descrever o estado mecânico de um sistema particular é mediante o desenvolvimento de um modelo matemático, e então, resolver este modelo com ajuda de métodos matemáticos ou analise numérica.

Seguindo este critério, o Método dos Elementos de Contorno [5], tem-se mostrado uma ferramenta poderosa e eficiente. $\mathrm{O}$ uso da Função de Green numérica [11] como solução fundamental evita a discretização do contorno da trinca quando este está descarregado, uma vez que esta solução, para força de superfície, é nula nas faces da trinca. Consequentemente, uma característica importante é tornar desnecessária a subdivisão da trinca em elementos de contorno.

O Método da Dupla Reciprocidade [4] permite resolver problemas transientes usando soluções fundamentais da estática, e que se revelou bastante adequado e eficaz na solução de problemas com ações no domínio. O objetivo do método é transformar a integral de domínio do termo 
existente, em uma integral de contorno. A estratégia é fazer a substituição da grandeza que tem as características de ação de domínio pelo produto de duas outras variáveis, ficando uma delas dependente das variáveis espaciais.

Desta forma, neste trabalho estuda-se o MEC, a Função de Green numérica e o método da Dupla Reciprocidade na resolução de problemas de mecânica da fratura com ações de domínio considerado como peso próprio [15].

Dois exemplos são apresentados, o primeiro corresponde a uma viga com trinca centralizada e carregamento reverso, e o segundo é uma viga com trinca centralizada e carregamento como peso próprio. Ambos os exemplos foram validados comparando-os com o trabalho de Karami e Kuhn [6].

\section{O MEC E A FUNÇÃO DE GREEN NUMÉRICA}

A Equação de Navier, que é uma equação de equilíbrio em termo de deslocamentos, é representada em um sistema 2-D como se ve na equação (2.1). As formulas são introduzidas empregando a notação indicial [16]:

$$
G u_{j, k k}+\frac{G}{1-2 v} u_{k, k j}=b_{j}
$$

onde $b_{j}$ representa o efeito de domínio (neste trabalho, peso próprio), $u$ é o deslocamento; $G$ e $v$ são constantes físicas do material.

O MEC [5] basicamente transforma equações diferenciais parciais, que governam o domínio do problema em equações integrais envolvendo valores de contorno, após a introdução de funções de ponderação denominadas soluções fundamentais.

Considera-se que existe continuidade de deslocamentos nos pontos de contorno $\xi$, desta forma a representação integral da componente $u_{j}$ de deslocamento é dada pela equação (2.2):

$$
\begin{aligned}
C_{i j}(\xi) u_{j}(\xi)+\int_{\Gamma} p_{i j}^{k}(\xi, x) u_{j}(x) d \Gamma(x)-\int_{\Gamma} u_{i j}^{k}(\xi, x) p_{j}(x) d \Gamma(x) & \\
& =\int_{\Omega} u_{i j}^{k}(\xi, x) b_{j}(x) d \Omega
\end{aligned}
$$

onde $\Gamma$ representa o contorno do problema; $u_{i j}^{k}$ e $p_{i j}^{k}$ na formulação tradicional correspondem à solução fundamental de Kelvin; $C_{i j}$ é um coeficiente relacionado à geometria do corpo e $\xi$ é o ponto fonte.

As soluções fundamentais para deslocamentos e forças de superfície são definidas como segue:

$$
u_{i j}^{k}(\xi, x)=\frac{-1}{8 \pi(1-v) G}\left\{(3-4 v) \ln (r) \delta_{i j}-r, i,,_{j}\right\}
$$

$\mathrm{e}$

$$
p_{i j}^{k}(\xi, x)=\frac{-1}{4 \pi(1-v) r}\left\{\left[(1-2 v) \delta_{i j}+2 r,{ }_{i} r, j\right] \frac{\partial r}{\partial n}-(1-2 v)\left(r_{i} n_{j}-r,{ }_{j} n_{i}\right)\right\}
$$

Neste ponto, para introduzir o conceito da Função de Green numérica [11], desconsidera-se o termo que corresponde à ação de domínio, sendo retomado na seguinte seção. 
As funções de Green para a solução de problemas da Mecânica da Fratura podem ser obtidas pela superposição de duas soluções parciais; a primeira corresponde à solução de Kelvin, enquanto que a segunda corresponde ao problema de uma placa infinita contendo uma trinca de comprimento $2 a$, com condição de contorno de forças prescritas ao longo de seu contorno e chamada de solução complementar, como pode-se observar na figura 1.

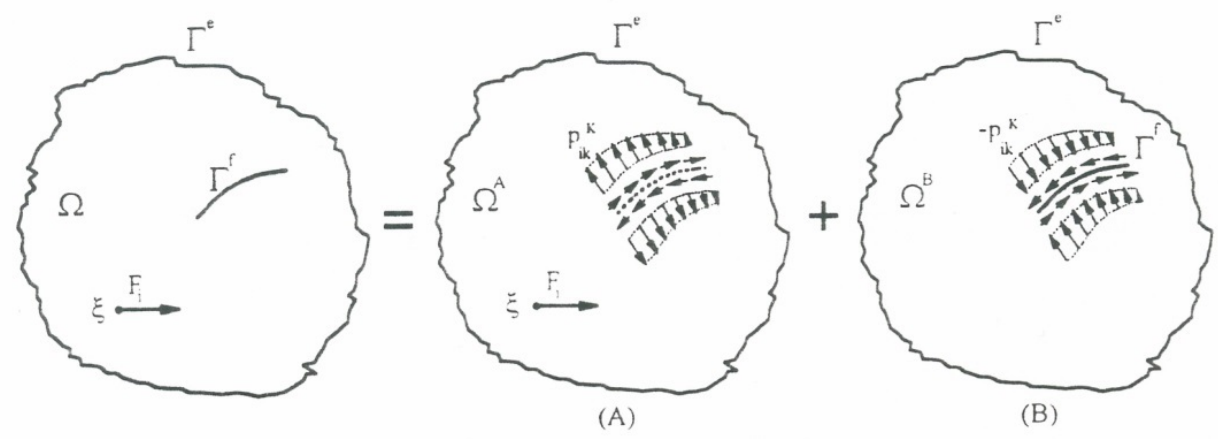

Figura 1: Carga Unitária $F_{i}$ aplicada num espaço infinito com trinca descarregada (A) Problema de Kelvin; (B) problema complementar.

Desta forma a Função de Green é representada pelas equações (2.5) e (2.6):

$$
\begin{aligned}
& u_{i j}^{*}(\xi, x)=u_{i j}^{k}(\xi, x)+u_{i j}^{c}(\xi, x) . \\
& p_{i j}^{*}(\xi, x)=p_{i j}^{k}(\xi, x)+p_{i j}^{c}(\xi, x) .
\end{aligned}
$$

onde $u_{i j}^{*}(\xi, x)$ e $p_{i j}^{*}(\xi, x)$ são respectivamente os deslocamentos e forças de superfície fundamentais, na direção $j$ no ponto campo $x$ devido a uma carga unitária aplicada no ponto fonte $\xi$ na direção $i$.

A solução de Kelvin, $u_{i j}^{k}(\xi, x)$ e $p_{i j}^{k}(\xi, x)$, é a tradicional do Método dos Elementos de Contorno, ficando para ser determinado o termo que corresponde à solução complementar $u_{i j}^{c}(\xi, x) \mathrm{e}$ $p_{i j}^{c}(\xi, x)$.

As deduções para a determinação das componentes de deslocamento e força de superfície da parte complementar da Função de Green são dadas em [13] e apresentadas a seguir.

A equação integral de contorno da equação (2.2) para um elemento que contém uma trinca é expressa por:

$$
\begin{aligned}
C_{i j}(\xi) u_{j}(\xi)=\int_{\Gamma^{E}} p_{j}(x) u_{i j}^{k}(\xi, x) & d \Gamma(x)-\int_{\Gamma^{E}} u_{j}(x) p_{i j}^{k}(\xi, x) d \Gamma(x) \\
& +\int_{\Gamma^{F}} p_{j}(x) u_{i j}^{k}(\xi, x) d \Gamma(x)-\int_{\Gamma^{F}} u_{j}(x) p_{i j}^{k}(\xi, x) d \Gamma(x) .
\end{aligned}
$$

onde $\Gamma^{E}$ é o contorno externo e $\Gamma^{F}$ é o contorno referente à superfície da trinca. As integrais com sinal negativo no segundo membro da equação (2.7) devem ser consideradas como valor principal de Cauchy de acordo com a posição do ponto fonte. 
A equação integral para forças de superfície é obtida por derivação da equação (2.7)em relação a $\xi$

$$
\begin{aligned}
p_{j}(\xi)=\int_{\Gamma^{E}} p_{j}(x) U_{i j}^{k}(\xi, x) d \Gamma(x) & -\int_{\Gamma^{E}} u_{j}(x) P_{i j}^{k}(\xi, x) d \Gamma(x) \\
& +\int_{\Gamma^{F}} p_{j}(x) U_{i j}^{k}(\xi, x) d \Gamma(x)-\int_{\Gamma^{F}} u_{j}(x) P_{i j}^{k}(\xi, x) d \Gamma(x) .
\end{aligned}
$$

onde $U_{i j}^{k}=\frac{\partial u_{i j}^{k}}{\partial \xi}$ e $P_{i j}^{k}=\frac{\partial p_{i j}^{k}}{\partial \xi}$.

As equações (2.7) e (2.8) satisfazem as condições de regularidade de radiação de tal forma que es integrais de contorno no infinito se anulam. Para simular a condição de trinca descarregada é necessário considerar a condição de contorno ao longo da trinca da solução B da figura 1, de tal forma que $p_{i k}^{c}(\xi, \zeta)=-p_{i k}^{k}(\xi, \zeta)$. Assim, fica definida a seguinte equação integral para o problema complementar:

$$
u_{i j}^{c}(\xi, x)=-\int_{\Gamma^{F}} u_{j k}^{k}(x, \zeta) p_{i k}^{k}(\xi, \zeta) d \Gamma(\zeta)-\int_{\Gamma^{F}} p_{j k}^{k}(x, \zeta) u_{i k}^{c}(\xi, \zeta) d \Gamma(\zeta) .
$$

Considerando que $S$ e $I$ correspondem à superfície superior e inferior da trinca $\left(\Gamma^{F}=\Gamma^{S}+\Gamma^{I}\right)$ é possível integrar somente sobre $\Gamma^{I}$, considerando que a força de superfície ao longo da superfície superior é de sinal contrário à da face inferior e que os deslocamento $u_{j k}^{k}(x, \zeta)$ são contínuos, o que fornece:

$$
\begin{aligned}
u_{i j}^{c}(\xi, x)=-\int_{\Gamma^{I}} u_{j k}^{k}(x, \zeta)\left[p_{i k}^{k}\left(\xi, \zeta^{S}\right)+\right. & \left.p_{i k}^{k}\left(\xi, \zeta^{I}\right)\right] d \Gamma(\zeta) \\
& +\int_{\Gamma^{I}} p_{j k}^{k}(x, \zeta)\left[u_{i k}^{c}\left(\xi, \zeta^{S}\right)-u_{i k}^{c}\left(\xi, \zeta^{I}\right)\right] d \Gamma(\zeta)
\end{aligned}
$$

O campo de tensões no contorno da trinca é contínuo: assim, as forças de superfície da primeira integral da equação (2.10) se anulam. Define-se a abertura da trinca $\left(c_{i k}(\xi, \zeta)\right)$ como a diferença entre o deslocamento do contorno superior $\Gamma^{S}$ e o inferior $\Gamma^{I}$

$$
c_{i k}(\xi, \zeta)=u_{i k}^{c}\left(\xi, \zeta^{S}\right)-u_{i k}^{c}\left(\xi, \zeta^{I}\right)
$$

Logo, pode-se escrever a seguinte equação

$$
u_{i j}^{c}(\xi, x)=\int_{\Gamma^{I}} p_{j k}^{k}(x, \zeta) c_{i k}(\xi, \zeta) d \Gamma(\zeta)
$$

E, analogamente, para força de superfície

$$
p_{i j}^{c}(\xi, x)=\int_{\Gamma^{I}} P_{j k}^{k}(x, \zeta) c_{i k}(\xi, \zeta) d \Gamma(\zeta) .
$$

As equações (2.12) e (2.13) ficam definidas se for conhecido o valor da abertura da trinca (equação (2.11)). Desta forma, o primeiro passo é determinar o valor de $c_{i k}(\xi, \zeta)$, resolvendo a equação integral hiper-singular de contorno que é gerada calculando o limite da equação (2.13) quando o ponto $x$ é deslocado até o contorno $\Gamma^{I}[12]$. Assim

$$
P F \int_{\Gamma^{I}} P_{j k}^{k}(\bar{\zeta}, \zeta) c_{i k}(\xi, \zeta) d \Gamma(\zeta)=-p_{i j}^{k}(\xi, \bar{\zeta})
$$


onde $P F$ indica a parte finita da integral.

A equação (2.14) pode ser resolvida pelo método dos resíduos ponderados, usando a técnica da colocação pontual. Assim, usando a função delta de Dirac para ponderar a equação num certo número de pontos $\bar{\zeta}_{m}(m=1, \ldots, M)$ sobre $\Gamma^{I}$, a seguinte equação é obtida,

$$
P F \int_{\Gamma^{I}} P_{j k}^{k}\left(\bar{\zeta}_{m}, \zeta\right) c_{i k}(\xi, \zeta) d \Gamma(\zeta)=-p_{i j}^{k}\left(\xi, \bar{\zeta}_{m}\right)
$$

Pode-se escrever e equação (2.15) utilizando um termo corretor $E_{i j}$ [2] e a integração numéria simples, como:

$$
\sum_{n=1}^{N}|J|_{n}\left(P_{j k}^{k}\left(\bar{\zeta}_{m}, \zeta_{n}\right) c_{i k}\left(\xi, \zeta_{n}\right) W_{n}\right)-E_{i j}=-p_{i j}^{k}\left(\xi, \bar{\zeta}_{m}\right) \quad, m=1,2, \ldots, M .
$$

onde $|J|_{n}$ é o Jacobiano da transformação para o intervalo de integração, $\zeta_{n}$ é o ponto da trinca correspondente ao ponto de Gauss $n, W_{n}$ é o peso associado a esse ponto e $N$ é o número total de pontos de integração.

O termo corretor $E_{i j}$ é introduzido para corrigir o resultado da integração numérica, devido às diferenças que ocorrem ao transformar a integral em parte finita em integral numérica [7].

O tratamento numérico da equação (2.16) se faz com a substituição das séries infinitas que definem as funções de Bessel por expressões polinomiais e logarítmicas que as interpolam por trechos. Desta forma o comportamento da parte imaginária é regular enquanto que a parte real da solução fundamental é singular em $r=0$ [1]. A integral à direita da equação (2.15) pode ser decomposta na seguinte forma:

$$
\begin{aligned}
P F \int_{\Gamma^{I}} P_{j k}^{k}\left(\bar{\zeta}_{m}, \zeta\right) & c_{i k}(\xi, \zeta) d \Gamma(\zeta)=P F \int_{\Gamma^{I}} P_{j k}^{K}\left(\bar{\zeta}_{m}, \zeta\right) c_{i k}(\xi, \zeta) d \Gamma(\zeta) \\
& +\int_{\Gamma^{I}} P_{j k}^{L o g}\left(\bar{\zeta}_{m}, \zeta\right) c_{i k}(\xi, \zeta) d \Gamma(\zeta)+\int_{\Gamma^{I}} P_{j k}^{R e g}\left(\bar{\zeta}_{m}, \zeta\right) c_{i k}(\xi, \zeta) d \Gamma(\zeta)
\end{aligned}
$$

Na equação(2.17), $P_{j k}^{K}$ é a solução hipersingular estática, derivada da solução de Kelvin, $P_{j k}^{L o g}$ são as parcelas de comportamento logarítmico e $P_{j k}^{R e g}$ são as parcelas de comportamento regular.

São analisadas as integrais do segundo membro da equação (2.17), tendo em vista a expansão de $c_{i k}$ em série de Taylor em relação ao ponto $\bar{\zeta}_{m}$.

$$
\begin{aligned}
c_{i k}(\xi, \zeta)=c_{i k}\left(\xi, \bar{\zeta}_{m}\right)+\frac{\partial c_{i k}\left(\xi, \bar{\zeta}_{m}\right)}{\partial x(\zeta)}(x(\zeta) & \left.-x\left(\bar{\zeta}_{m}\right)\right) \\
& +\sum_{n=2}^{\infty} \frac{1}{n !} \frac{\partial^{n} c_{i k}\left(\xi, \bar{\zeta}_{m}\right)}{\partial x^{n}(\zeta)}\left(x(\zeta)-x\left(\bar{\zeta}_{m}\right)\right)^{n}
\end{aligned}
$$


Assim, para o termo definido por $P_{j k}^{K}$, pode-se estabelecer uma parcela do erro da equação (2.16) através da seguinte igualdade,

$$
\begin{gathered}
\sum_{n=1}^{N}|J|_{n}\left(P_{j k}^{K}\left(\bar{\zeta}_{m}, \zeta_{n}\right) c_{i k}\left(\xi, \zeta_{n}\right) W_{n}\right)-P F \int_{-a}^{a} P_{j k}^{K}\left(\bar{\zeta}_{m}, \zeta\right) c_{i k}(\xi, \zeta) d \Gamma(\zeta) \\
=c_{i k}\left(\xi, \bar{\zeta}_{m}\right)\left[\sum_{n=1}^{N}|J|_{n}\left(P_{j k}^{K}\left(\bar{\zeta}_{m}, \zeta_{n}\right) c_{i k}\left(\xi, \zeta_{n}\right) W_{n}\right)-P F \int_{-a}^{a} P_{j k}^{K}\left(\bar{\zeta}_{m}, \zeta\right) c_{i k}(\xi, \zeta) d \Gamma(\zeta)\right] \\
+\frac{\partial c_{i k}\left(\xi, \bar{\zeta}_{m}\right)}{\partial x(\zeta)}\left[\sum_{n=1}^{N}|J|_{n}\left(\left(\zeta_{n}-\zeta_{m}\right) P_{j k}^{K}\left(\bar{\zeta}_{m}, \zeta_{n}\right) c_{i k}\left(\xi, \zeta_{n}\right) W_{n}\right)\right. \\
\left.-V P \int_{-a}^{a}\left(\zeta_{n}-\zeta_{m}\right) P_{j k}^{K}\left(\bar{\zeta}_{m}, \zeta\right) c_{i k}(\xi, \zeta) d \Gamma(\zeta)\right]
\end{gathered}
$$

onde VP refere-se ao valor principal de Cauchy. Para o contorno inferior da trinca $\Gamma^{I}$, o intervalo de integração corresponde ao comprimento da trinca $2 a$ ou $[-a, a]$.

Esta mesma expressão pode ser escrita de forma mais compacta como segue:

$$
\begin{aligned}
& \sum_{n=1}^{N}|J|_{n}\left(P_{j k}^{K}\left(\bar{\zeta}_{m}, \zeta_{n}\right) c_{i k}\left(\xi, \zeta_{n}\right) W_{n}\right)-P F \int_{-a}^{a} P_{j k}^{K}\left(\bar{\zeta}_{m}, \zeta\right) c_{i k}(\xi, \zeta) d \Gamma(\zeta) \\
& c_{i k}\left(\xi, \bar{\zeta}_{m}\right) e_{j k}^{K 1}+\frac{\partial c_{i k}\left(\xi, \bar{\zeta}_{m}\right)}{\partial x(\zeta)} e_{j k}^{K 2}
\end{aligned}
$$

onde os termos $e_{j k}^{K 1}$ e $e_{j k}^{K 2}$ são dados por

$$
\begin{aligned}
e_{j k}^{K 1} & =2\left\{a \sum_{n=1}^{N}\left(\frac{W_{n}}{\left(\zeta_{n}-\bar{\zeta}_{m}\right)^{2}}\right)-\left(\frac{-2 a}{a^{2}-\bar{\zeta}_{m}^{2}}\right)\right\} . \\
e_{j k}^{K 2} & =2\left\{a \sum_{n=1}^{N}\left(\frac{W_{n}}{\zeta_{n}-\bar{\zeta}_{m}}\right)-\ln \left(\frac{a-\bar{\zeta}_{m}}{a+\bar{\zeta}_{m}}\right)\right\} .
\end{aligned}
$$

Analogamente, o erro devido à singularidade logarítmica em relação à integração pela quadratura de Gauss se deve exclusivamente ao primeiro termo da série

$$
\begin{array}{r}
\sum_{n=1}^{N}|J|_{n}\left(P_{j k}^{\log }\left(\bar{\zeta}_{m}, \zeta_{n}\right) c_{i k}\left(\xi, \zeta_{n}\right) W_{n}\right)-P F \int_{-a}^{a} P_{j k}^{L o g}\left(\bar{\zeta}_{m}, \zeta\right) c_{i k}(\xi, \zeta) d \Gamma(\zeta) \\
=c_{i k}\left(\xi, \bar{\zeta}_{m}\right)\left[\sum_{n=1}^{N}|J|_{n}\left(P_{j k}^{L o g}\left(\bar{\zeta}_{m}, \zeta_{n}\right) c_{i k}\left(\xi, \zeta_{n}\right) W_{n}\right)\right. \\
\left.-P F \int_{-a}^{a} P_{j k}^{L o g}\left(\bar{\zeta}_{m}, \zeta\right) c_{i k}(\xi, \zeta) d \Gamma(\zeta)\right]
\end{array}
$$

E pode ser escrito na forma compacta como segue,

$$
\begin{aligned}
\sum_{n=1}^{N}|J|_{n}\left(P_{j k}^{L o g}\left(\bar{\zeta}_{m}, \zeta_{n}\right) c_{i k}\left(\xi, \zeta_{n}\right) W_{n}\right)-P F \int_{-a}^{a} P_{j k}^{L o g}\left(\bar{\zeta}_{m}, \zeta\right) c_{i k}(\xi, \zeta) d \Gamma(\zeta) & =c_{i k}\left(\xi, \bar{\zeta}_{m}\right) e_{j k}^{L o g}
\end{aligned}
$$


onde a expressão de $e_{j k}^{\log }$ é dada por

$$
\begin{aligned}
e_{j k}^{L o g}=s^{2}\left\{a \sum_{n=1}^{N} W_{n} \ln \left(\left|\zeta_{n}-\bar{\zeta}_{m}\right|\right)\right. & \\
& {\left.\left[\left(\bar{\zeta}_{m}-a\right) \ln \left(\left|\bar{\zeta}_{m}-a\right|\right)+\left(\left|\bar{\zeta}_{m}+a\right|\right) \ln \left(\left|\bar{\zeta}_{m}+a\right|\right)-2 a\right]\right\} }
\end{aligned}
$$

Uma vez que só se está considerando o erro referente às integrais singulares pode-se escrever,

$$
\sum_{n=1}^{N}|J|_{n}\left(P_{j k}^{R e g}\left(\bar{\zeta}_{m}, \zeta_{n}\right) c_{i k}\left(\xi, \zeta_{n}\right) W_{n}\right)-P F \int_{-a}^{a} P_{j k}^{R e g}\left(\bar{\zeta}_{m}, \zeta\right) c_{i k}(\xi, \zeta) d \Gamma(\zeta)=0
$$

Assim, a integral da equação (2.15) é aproximada como:

$$
\begin{aligned}
P F \int_{-a}^{a} P_{j k}^{k}\left(\bar{\zeta}_{m}, \zeta\right) c_{i k}(\xi, \zeta) d \Gamma(\zeta)=\sum_{n=1}^{N}|J|_{n}\left[P_{j k}^{k}\left(\bar{\zeta}_{m}, \zeta_{n}\right) c_{i k}\left(\xi, \zeta_{n}\right) W_{n}\right] & \\
& -\left[c_{i k}\left(\xi, \bar{\zeta}_{m}\right)\left(e_{j k}^{K 1}+e_{j k}^{L o g}\right)+\frac{\partial c_{i k}\left(\xi, \bar{\zeta}_{m}\right)}{\partial x(\zeta)} e_{j k}^{K 2}\right]
\end{aligned}
$$

E o termo corretor é, portanto

$$
E_{i j}=c_{i k}\left(\xi, \bar{\zeta}_{m}\right)\left(e_{j k}^{K 1}+e_{j k}^{L o g}\right)+\frac{\partial c_{i k}\left(\xi, \bar{\zeta}_{m}\right)}{\partial x(\zeta)} e_{j k}^{K 2} .
$$

Fazendo o número de pontos de colocação coincidir com o número de pontos usados para a integração numérica $(M=N)$, reescreve-se a equação (2.16):

$$
a \sum_{n=1}^{N}\left(P_{j k}^{k}\left(\bar{\zeta}_{m}, \zeta_{n}\right) c_{i k}\left(\xi, \zeta_{n}\right) W_{n}\right)-E_{i j}=-p_{i j}^{k}\left(\xi, \bar{\zeta}_{m}\right) \quad, m=1,2, \ldots, N .
$$

Como $n=m$, surgem indeterminações que são resolvidas calculando-se o processo de limite quando $\bar{\zeta}_{m} \rightarrow \zeta_{n}$, obtendo-se,

$$
\begin{gathered}
\lim _{\bar{\zeta}_{m} \rightarrow \zeta_{n}}\left\{\frac{1}{\left(\zeta_{n}-\zeta_{m}\right)^{2}}\left[c_{i k}\left(\xi, \zeta_{n}\right)-c_{i k}\left(\xi, \bar{\zeta}_{m}\right)-\left(\zeta_{n}-\bar{\zeta}_{m}\right) \frac{\partial c_{i k}\left(\xi, \bar{\zeta}_{m}\right)}{\partial \zeta}\right]\right\}= \\
\lim _{\bar{\zeta}_{m} \rightarrow \zeta_{n}}\left\{c_{i k}\left(\xi, \zeta_{n}\right)-c_{i k} \ln \left(\left|\zeta_{n}-\bar{\zeta}_{m}\right|\right)\right\}=0
\end{gathered}
$$


Finalmente

$$
\begin{gathered}
a \sum_{\substack{n=1 \\
n \neq m}}^{N}\left(P_{j k}^{k}\left(\bar{\zeta}_{m}, \zeta_{n}\right) c_{i k}\left(\xi, \zeta_{n}\right) W_{n}\right)+c_{i k}\left(\xi, \bar{\zeta}_{m}\right) P_{j k}^{R e g}\left(\bar{\zeta}_{m}, \zeta_{n}\right) \\
-c_{i k}\left(\xi, \bar{\zeta}_{m}\right)\left\{2\left[a \sum_{\substack{n=1 \\
n \neq m}}^{N}\left(\frac{W_{n}}{\left(\zeta_{n}-\zeta_{m}\right)^{2}}\right)+\frac{2 a}{a^{2}-\bar{\zeta}_{m}^{2}}\right]+\right. \\
\left.s^{2}\left[a \sum_{\substack{n=1 \\
n \neq m}}^{N}\left(W_{n} \ln \left(\zeta_{n}-\bar{\zeta}_{m}\right)\right)+2 a-\left(\left(\left|\bar{\zeta}_{m}-a\right|\right) \ln \left(\left|\bar{\zeta}_{m}-a\right|\right)+\left(\left|\bar{\zeta}_{m}+a\right|\right) \ln \left(\left|\bar{\zeta}_{m}+a\right|\right)\right)\right]\right\} \\
-\frac{\partial c_{i k}\left(\xi, \bar{\zeta}_{m}\right)}{\partial x(\zeta)}\left\{2\left[a \sum_{\substack{n=1 \\
n \neq m}}^{N}\left(\frac{W_{n}}{\zeta_{n}-\bar{\zeta}_{m}}\right)-\ln \left(\frac{a-\bar{\zeta}_{m}}{a+\bar{\zeta}_{m}}\right)\right]\right\} \\
+a W_{n} \frac{\partial^{2} c_{i k}\left(\xi, \bar{\zeta}_{m}\right)}{\partial \zeta^{2}}=-p_{i j}^{k}\left(\xi, \bar{\zeta}_{m}\right) \quad m=1,2, \ldots, N .
\end{gathered}
$$

Com a finalidade de calcular as derivadas de $c_{i k}(\xi, \zeta)$, adota-se a interpolação polinomial de Lagrange. Uma vez que o comportamento das aberturas é proporcional a $\sqrt{\rho}$, onde $\rho$ é a distância até a ponta da trinca, define-se:

$$
c_{i k}(\xi, \zeta) \approx \sqrt{1-\eta^{2}} \sum_{n=1}^{N} D_{n}(\eta) \frac{c_{i k}\left(\xi, \zeta_{n}\right)}{\sqrt{1-\eta_{n}^{2}}} .
$$

onde $\eta=\frac{\zeta}{a}$ é a coordenada natural da trinca e $\eta_{n}=\frac{\zeta_{n}}{a}$

$$
D_{n}(\eta)=\prod_{\substack{t=1 \\ t \neq n}}^{T} \frac{\left(\eta-\eta_{t}\right)}{\eta_{n}-\eta_{t}}
$$

onde $D_{n}$ é um polinômio de Lagrange de ordem $(N-1)$, $T$ é um número inteiro positivo igual a $N$.

Assim, a primeira derivada é,

$$
\begin{aligned}
& \frac{\partial c_{i k}(\xi, \zeta)}{\partial \zeta}=\frac{\partial c_{i k}(\xi, \zeta)}{\partial \eta} \frac{\partial \eta}{\partial \zeta} \\
& \frac{\partial c_{i k}(\xi, \zeta)}{\partial \zeta} \cong \frac{1}{a} \frac{-\eta}{\sqrt{1-\eta^{2}}} \sum_{n=1}^{N} D_{n}(\eta) \frac{c_{i k}\left(\xi, \zeta_{n}\right)}{\sqrt{1-\eta_{n}^{2}}}+\frac{\sqrt{1-\eta^{2}}}{a} \sum_{n=1}^{N} D_{n}^{\prime}(\eta) \frac{c_{i k}\left(\xi, \zeta_{n}\right)}{\sqrt{1-\eta_{n}^{2}}}
\end{aligned}
$$


E a segunda derivada é,

$$
\begin{aligned}
& \frac{\partial^{2} c_{i k}(\xi, \zeta)}{\partial \zeta^{2}}=\frac{\partial}{\partial \eta}\left[\frac{\partial c_{i k}(\xi, \zeta)}{\partial \zeta}\right] \frac{\partial \eta}{\partial \zeta} \\
& \begin{aligned}
\frac{\partial^{2} c_{i k}(\xi, \zeta)}{\partial \zeta^{2}}=-\frac{1}{a^{2}\left(1-\eta^{2}\right)^{3 / 2}} \sum_{n=1}^{N} D_{n}(\eta) \frac{c_{i k}\left(\xi, \zeta_{n}\right)}{\sqrt{1-\eta_{n}^{2}}}-\frac{2 \eta}{a^{2} \sqrt{1-\eta^{2}}} \sum_{n=1}^{N} D_{n}^{\prime}(\eta) \frac{c_{i k}\left(\xi, \zeta_{n}\right)}{\sqrt{1-\zeta_{n}^{2}}} \\
\quad+\frac{\sqrt{1-\eta^{2}}}{a^{2}} \sum_{n=1}^{N} D_{n}^{\prime \prime}(\eta) \frac{c_{i k}\left(\xi, \zeta_{n}\right)}{\sqrt{1-\eta_{n}^{2}}}
\end{aligned}
\end{aligned}
$$

A equação (2.32) forma um sistema matricial de $4 N$ equações subdivididas em dois subsistemas, de dimensão $2 N$, compartilhando a mesma matriz quadrada, que são usados para a determinação das aberturas fundamentais da trinca nos pontos de Gauss. O primeiro, para $i=1$, com as incógnitas $c_{l k}\left(\xi, \zeta_{n}\right)$ e o termo independente $p_{l j}^{k}\left(\xi, \bar{\zeta}_{m}\right)$; e o segundo, para $i=2$, com as incógnitas $c_{2 k}\left(\xi, \zeta_{n}\right)$ e o termo independente $p_{2 j}^{k}\left(\xi, \bar{\zeta}_{m}\right)$. Assim, pode-se escrever na forma matricial:

$$
S c_{i}(\xi)=p_{i}^{k}(\xi)
$$

É importante notar que a matriz $S$ é função da geometria da trinca e permanece constante para qualquer posição do ponto fonte, não aumentando o tamanho do sistema a ser resolvido com o refinamento da discretização do contorno do problema.

Das equações (2.5), (2.6) e equações (2.12), (2.13), pode-se escrever a Função de Green numérica para uma única trinca da forma seguinte:

$$
\begin{aligned}
& u_{i j}^{*}(\xi, x)=u_{i j}^{k}(\xi, x)+\sum_{n=1}^{N}|J|_{n} p_{j k}^{k}\left(x, \zeta_{n}\right) c_{i k}\left(\xi, \zeta_{n}\right) W_{n} . \\
& p_{i j}^{*}(\xi, x)=p_{i j}^{k}(\xi, x)+\sum_{n=1}^{N}|J|_{n} P_{j k}^{k}\left(x, \zeta_{n}\right) c_{i k}\left(\xi, \zeta_{n}\right) W_{n} .
\end{aligned}
$$

Finalmente, a equação integral de contorno para o problema da mecânica da fratura é escrita na forma

$$
C_{i j} u_{j}(\xi)=\int_{\Gamma^{E}} p_{j}(x) u_{i j}^{*}(\xi, x) d \Gamma(x)-\int_{\Gamma^{E}} u_{j}(x) p_{i j}^{*}(\xi, x) d \Gamma(x) .
$$

\section{O MÉTODO DA DUPLA RECIPROCIDADE}

Na equação (2.2) tem-se a equação integral de contorno com uma integral de domínio na qual se vai aplicar os conceitos da Dupla Reciprocidade para transformar esta numa integral de contorno. Segundo a filosofia do Método da Dupla Reciprocidade o termo de domínio é aproximado por um somatório de funções na forma indicial seguinte (equação (3.1)):

$$
b_{j}(x)=\alpha_{j}^{m} f^{m}=\alpha_{j}^{1} f^{1}+\alpha_{j}^{2} f^{2}+\ldots+\alpha_{j}^{n} f^{n} .
$$


onde os pontos $m$ são pontos de colocação, $f$ é uma função de aproximação e $\alpha$ é um conjunto de coeficientes iniciais desconhecidos.

Substituindo a equação (3.1) na integral de domínio da equação (2.2) tem-se a seguinte expressão:

$$
\int_{\Omega} u_{i j}^{*}(\xi, x) b_{j}(x) d \Omega=\alpha_{j}^{m} \int_{\Omega} f^{m}(x) u_{i j}^{*}(\xi, x) d \Omega .
$$

Para transformar o lado direito da expressão anterior numa integral de contorno, é necessário rescrever $f^{m}(x)$ em termos de uma função auxiliar $\psi_{n j}^{m}$ que precisa satisfazer à equação de Navier, equação (2.1), com o termo de domínio sendo concentrada no domínio como se mostra a seguir:

$$
G \psi_{n j, i i}^{m}+\frac{G}{1-2 v} \psi_{n i, i j}^{m}=\delta_{n j} f^{m}
$$

Desta forma, substituindo a equação (3.3) na equação (3.2), e verificando que, por analogia, as mesmas operações já desenvolvidas na formulação tradicional podem ser empregadas, obtém-se a equação (3.4):

$$
C_{i j}^{l} u_{j}^{l}+\int_{\Gamma} p_{i j}^{*} u_{j} d \Gamma-\int_{\Gamma} u_{i j}^{*} p_{j} d \Gamma=\left(C_{i j}^{l} \psi_{n j}^{l m}+\int_{\Gamma} p_{i j}^{*} \psi_{n j}^{m} d \Gamma-\int_{\Gamma} u_{i j}^{*} \eta_{n j}^{m} d \Gamma\right) \alpha_{m}^{n}
$$

Nesta expressão o termo $\eta_{n j}^{m}$ corresponde às forças de superfície recíprocas, referentes à função auxiliar de interpolação $\psi_{n j}^{m}$, correspondente aos deslocamentos.

$$
\psi_{i j}^{m}=\frac{1}{30(1-v) \mu}\left[\left(3-\frac{10 v}{3}\right) \delta_{i j}-r_{i} r_{j}\right] r^{3}
$$

$\mathrm{e}$

$$
\eta_{i j}^{m}=\frac{1}{15(1-v)}\left[(4-5 v) r_{i} n_{j}-(1-5 v) r,{ }_{j} n_{i}+\left[(4-5 v) \delta_{i j}-r,{ }_{j} r, i\right] \frac{\partial r}{\partial n}\right] r^{2}
$$

onde $\frac{\partial r}{\partial n}=n_{i} r, i$

A equação (3.4) é discretizada dividindo-se o contorno em elementos e escrevendo-se para todos os nós $i$ em termos de matrizes globais. Chega-se então a um sistema geral de equações dada pela equação (3.7):

$$
\mathbf{H u}-\mathbf{G p}=(\mathbf{H} \psi-\mathbf{G} \eta) \mathbf{f}^{-\mathbf{1}} \mathbf{b} .
$$

A função $f^{m}$ escolhida é a função linear $r$ que quantifica a distância euclidiana entre dois pontos e que é amplamente usada na literatura.

\section{EXEMPLOS DE APLICAÇÃO}

\subsection{Viga com trinca centralizada e carregamento reverso}

De forma a comparar a formulação desenvolvida pelos autores é estudado um problema onde a ação de domínio é o peso próprio mas em sentido reverso, ou seja seguindo a direção positiva do eixo $y$, aplicado a uma viga bi-apoiada com trinca centrada. 
O parâmetro mais utilizado no estudo da Mecânica da Fratura Linear Elástica é o fator de intensidade de tensão $(K)$ que quando comparado a um valor crítico, determinado experimentalmente, permite prever o comportamento da trinca e, em consequência, a vida útil do componente trincado. Normalmente $K$ é função da forma e tamanho da trinca, tipo de carregamento e configuração da geometria do componente estrutural. Usualmente o fator de intensidade de tensão é escrito da seguinte forma: $K=Y \sigma \sqrt{\pi a}$ onde $\sigma$ é a tensão; $a$, uma medida relacionada ao comprimento de trinca; $Y$, uma função adimensional da geometria da trinca. $K_{0}=\sigma \sqrt{\pi a}$ corresponde ao fator de intensidade de tensão de uma placa infinita carregada remotamente, contendo uma trinca de comprimento $2 a$.

Neste exemplo, estuda-se uma chapa retangular com uma trinca de bordo reta e centralizada com carregamento vertical positivo, bi-apoiada, como é mostrado na figura 2 [6] [15]. Adotando um material linearmente elástico com as seguintes propriedades: módulo de Young igual a 1000; coeficiente de Poisson igual a 0,3, densidade igual à unidade e trinca de tamanhos 7 e 8 , $\operatorname{com} a / w=0.7$ e $h / w=4$. A discretização tem 197 nós no contorno, 96 elementos quadráticos e 40 pontos internos. Os valores de $h$ e $w$ correspondem ao comprimento e altura da viga respectivamente. Considera-se que a largura é unitária.

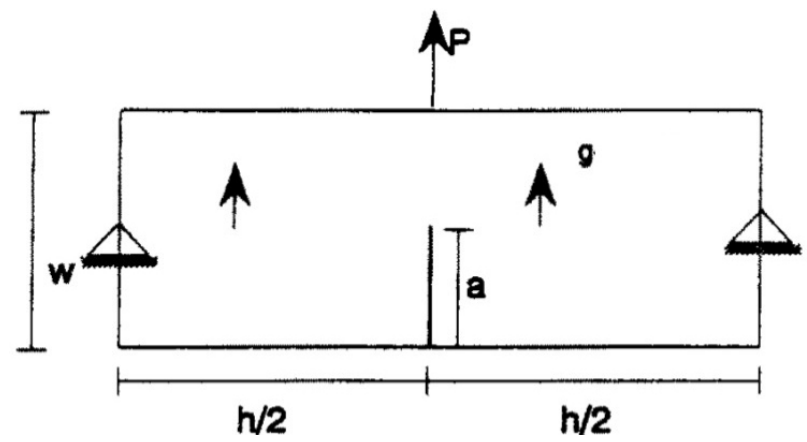

Figura 2: Chapa com carregamento reverso.

Tabela 1: Valores de $K_{l} / K_{0}$.

\begin{tabular}{cccc}
\hline$h / w$ & $a / w$ & $K_{l} / K_{0}$ & $K_{l} / K_{0}$ (Karami [6]) \\
\hline 2 & 0.7 & 0.27 & 0.216 \\
2 & 0.8 & 0.61 & 0.625 \\
3 & 0.7 & 0.81 & 0.717 \\
3 & 0.8 & 1.30 & 1.343 \\
4 & 0.7 & 1.44 & 1.222 \\
4 & 0.8 & 2.23 & 2.264 \\
\hline
\end{tabular}

Ao implementar os dados no programa, observa-se após a carga reversa ser aplicada, uma variação no Fator de Intensidade de Tensão (FIT), tabela 1, que também resultou em uma variação 
da trinca no eixo $y$, representado na tabela 2 , os dados tabelados descrevem uma superfície similar ao obtido na figura 3 [6].

Tabela 2: Valores da Abertura da Trinca.

\begin{tabular}{cc}
\hline Nó & $C_{y}$ \\
\hline 11 & 0.173309091 \\
12 & 1.488407728 \\
13 & 11.59123329 \\
14 & 10.30515245 \\
15 & 8.209602971 \\
16 & 6.809388777 \\
17 & 5.497799936 \\
18 & 4.451916365 \\
19 & 3.487847496 \\
20 & 2.692714304 \\
21 & 1.971155669 \\
22 & 1.376407442 \\
23 & 0.829984825 \\
24 & 0.375531989 \\
\hline
\end{tabular}

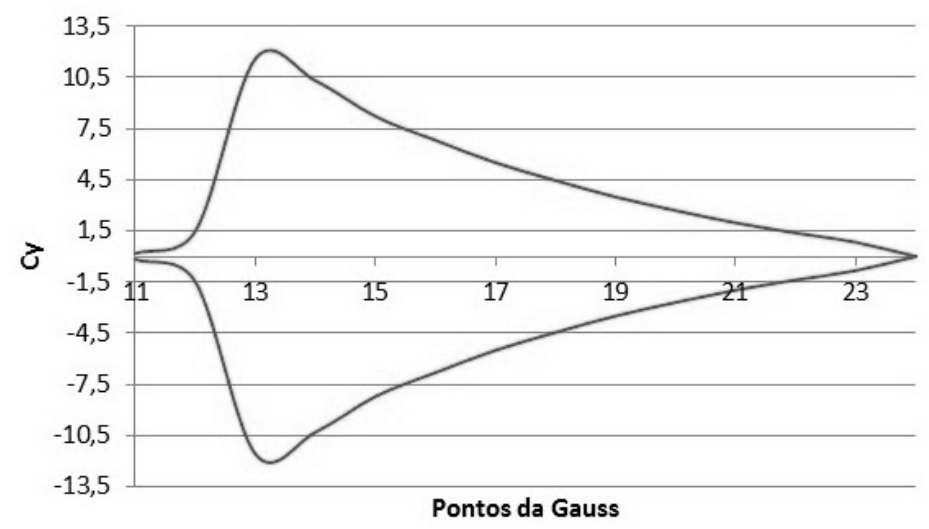

Figura 3: Gráfico da Abertura da Trinca obtido com a formulação MEC-FGN-DR.

\subsection{Viga com trinca centralizada e carregamento como peso próprio}

Este exemplo compara a formulação proposta da Mecânica da Fratura com o Método dos Elementos de Contorno e a função de Green numérica, analisando dois problemas específicos, onde o primeiro tem ação de domínio e o segundo uma força aplicada equivalente, ou seja a primeira 
utiliza a formulação da Dupla Reciprocidade e a outra não. O problema é resolvido por Karami and Kuhn [6] e os resultados numéricos para o caso de força aplicada são comparados com os valores teóricos de Tada et al. [10]. O exemplo é resolvido para ação de domínio como peso próprio e, também, para uma força aplicada pontual de valor equivalente ao peso próprio (onde é a densidade, $\mathrm{V}$ é o volume da viga e g é a constante gravitacional) na direção da gravidade e aplicado no ponto meio [14].

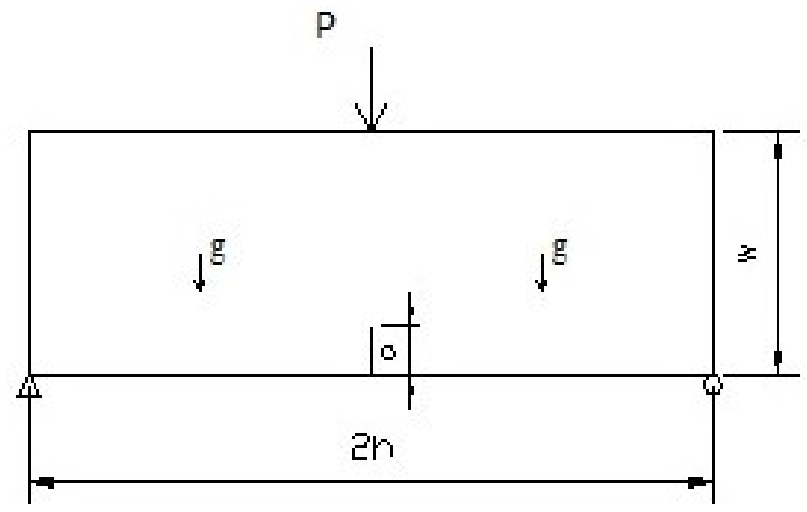

Figura 4: Viga com trinca centralizada e carregamento como peso próprio.

Estudam-se relações $a / w$ iguais a $0.1 ; 0.2 ; 0.3 ; 0.4$ e 0.5 . A geometria do problema é mostrado na figura 4 e para a análise numérica considera-se $\rho g=1$; coeficiente de Poisson ( $v$ ) é igual a 0.3; módulo de Young igual a 1000 e assume-se estado plano de deformações.

Os resultados estão resumidos na tabela 3. Observa-se que os valores obtidos com o peso próprio sempre são menores que os calculados com a força concentrada equivalente. a relação entre estes resultados é mantida em cerca de de $1 / 2$ e a explicação para isto é que o momento produzido pelo peso próprio é a metade do produzido pela força concentrada.

Na figura 5 estão plotados os resultados apresentados pela tabela 3, observa-se uma variação anormal nos resultados para a relação $a / w=0.4$, possivelmente por um erro numérico para este exemplo específico.

\section{CONCLUSÕES}

Neste trabalho foi estudado o Método dos Elementos de Contorno com a Função de Green Numérica e a Dupla Reciprocidade para a resolução de problemas da mecânica da fratura linear elástica numa chapa retangular com trinca reta de bordo sob uma carga de domínio do tipo reversa à força da gravidade. $\mathrm{O}$ estudo da trinca reta de bordo requer alguns cuidados pela dificuldade em modelar as duas superfícies da trinca e também por estar no contorno de problema, apresentando singularidades numéricas importantes. Os valores da abertura da trinca foram utilizados 
Tabela 3: FIT $\left(K_{l} / \rho g w \sqrt{\pi a}\right)$ para a viga com carga concentrada e peso próprio.

\begin{tabular}{cccccc}
\hline$a / w$ & Teórico & Teste $^{1}$ & Teste $^{2}$ & Razão & Teste $^{3}$ \\
\hline 0,1 & 24.17 & 24.88 & 12.55 & 0.50 & 13.52 \\
0,2 & 23.71 & 24.38 & 12.85 & 0.53 & 14.25 \\
0,3 & 25.07 & 25.59 & 13.31 & 0.52 & 15.01 \\
0,4 & 28.28 & 26.69 & 13.35 & 0.50 & 16.45 \\
0,5 & 33.99 & 33.42 & 17.53 & 0.52 & 19.23
\end{tabular}

${ }^{1}$ Método dos Elementos de Contorno com Carga Concentrada e Função de Green.

${ }^{2}$ Método dos Elementos de Contorno com Peso Próprio, Dupla Reciprocidade e Função de Green.

${ }^{3}$ Método dos Elementos de Contorno com Carga Equivalente e Função de Green.

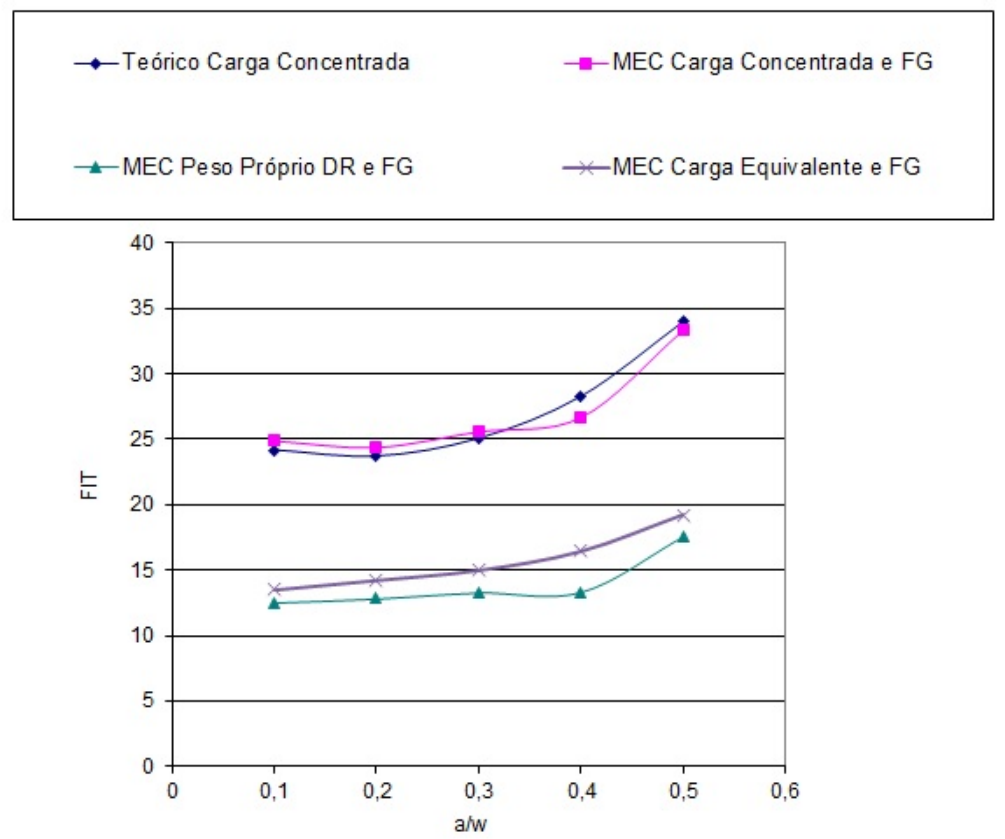

Figura 5: Variação do Fator de Intensidade de Tensões vs Comprimento da Trinca.

para observar o comportamento das superfícies da trinca. Os valores de $K_{l} / K_{0}$ foram calculados e comparados com a referência [6], sendo que os valores obtidos ficaram muito próximos.

No segundo exemplo da Viga com trinca centralizada e carregamento como peso próprio pode-se observar que os valores obtidos para diferentes relações de $\frac{a}{w}$ estão muito próximos dos valores 
teóricos. Segundo a referência [14] a relação entre o carregamento distribuído como peso próprio e uma carga equivalente concentrada deve ser igual a 0.5 e os resultados obtidos confirmam está afirmação.

Trabalhos futuros estão relacionados com o estudo de trincas inclinadas, problemas dinâmicos e problemas com multiplas trincas. Estudar o programa desenvolvido pelos autores, observando o tempo de processamento computacional é importante devido que ao trabalhar com um volume muito grande de entradas implica em processamentos longos e pode-se pensar em implementar códigos para paralelizar o programa, como o OpenMP, por exemplo.

\begin{abstract}
In recent years, the Boundary Element Method (MEC) has been applied successfully to mechanical problems of linear elastic fracture mechanic (LEFM) involving static and dynamic cases. To solve problems with domain actions (for example: gravitational forces, transient problems with speeds and accelerations, etc.) via BEM, Nardini and Brebbia presented in 1982 the Technique of the Dual Reciprocity (DR), initially with the intention of solving transient problems using static, but has proved to be quite adequate and effective in solving problems with actions of domains. Based on the above, this paper presents complementary studies initiated by Vera-Tudela in 2003, using the Technique of Numerical Green's Functions (FGN), together with the Dual Reciprocity. The development of these three formulations for the analysis of mechanical problems fractures under the effect of domain charges constitute a step forward in addressing the problems BEM. Although the use of Dual Reciprocity technique in fracture mechanics problems is not recent, their joint implementation with the Numerical Green Function represents a novelty in research and with the advantage of not having to do the discretization of the surfaces of the cracks because the integrations are made only on the contour of the problem. Two examples are presented, and the results compared with the reference literature and with the theory show and efficiency of the formulation.
\end{abstract}

Keywords: Boundary Element Method, Numerical Green’s Function, Dual Reciprocity.

\title{
REFERÊNCIAS
}

[1] L.P.S. Barra. "Aplicação do MEC à Mecânica da Fratura Elastodinâmica com Funções de Green Numéricas”. Ph.D. thesis, COPPE, UFRJ, Rio de Janeiro, RJ (1996).

[2] L.P.S. Barra \& J.C.F. Telles. A hyper-singular numerical Green's funtion generation for BEM applied to dynamic SIF problems. Engineering Analysis with Boundary Elements, (23) (1995), 77-87.

[3] G.E. Blandford, A.R. Ingraffea \& J.A. Liggett. Two-dimensional Stress Intensity Factor Computation using the Boundary Element Method. International Journal for Numerical Methods in Engineering, (17) (1981), 387-404.

[4] C.A. Brebbia, P.W. Partridge \& L.C. Wrobel. "The Dual Reciprocity Boundary Element Method". Computational Mechanics Publications, Southampton (1992).

[5] C.A. Brebbia, J.C.F. Telles \& L.C. Wrobel. "Boundary Elements Techniques: Theory and Applications in Engineering”. Springer-Verlag, London (1984). 
[6] G. Karami \& G. Kuhn. Body-Force Linear Elastic Stress Intensity Factor Calculation Using Boundary Element Method. Computer and Structures, (49) (1993), 107-115.

[7] H.R. Kutt. The numerical evaluation of principal value integrals by finite-part integration. Numerische Mahtematik, (24) (1975), 205-210.

[8] L.F. Martha, L.J. Gray \& A.R. Ingraffea. Three dimensional fracture simulation with a single domain, direct boundary element formulation. International Journal for Numerical Methods in Engineering, (35) (1992), 1907-1921.

[9] M.D. Snyder \& T.A. Cruse. Boundary integral equation analysis of cracked anisotropic plates. International Journal of Fracture, (11) (1975), 315-328.

[10] H. Tada, P.C. Paris \& G.R. Irwin. "The Stress Analysis of Cracks Handbook". Del Research Corporation, Hellertown (1983).

[11] J.C.F. Telles, G.S. Castor \& S. Guimarães. A Numerical Green's Function approach for boundary elements applied to fracture mechanics. International Journal for Numerical Methods in Engineering, (38) (1995), 3259-3274.

[12] J.C.F. Telles \& S. Guimarães. On the hyper-singular boundary element formulation for fracture mechanics applications. Engineering Analysis with Boundary Elements, (13) (1994), 353-363.

[13] J.C.F. Telles \& S. Guimarães. On the use of the Numerical Green's Function for SIF computations with Boundary Elements. In "First International Conference on Boundary Element Techniques". London (1999).

[14] S.P. Timoshenko \& J.N. Goodier. "Theory of Elasticity”. Mc Graw-Hill, New York (1970).

[15] C.A.R. Vera-Tudela. "Formulações Alternativas do MEC para Problemas Elastodinâmicos de Mecânica da Fratura com o uso da Função de Green Numérica". Ph.D. thesis, COPPE, UFRJ, Rio de Janeiro (2003).

[16] L.C. Wrobel. "The Boundary Element Method: Applications in Thermo-Fluids and Acoustic". John Wiley and Sons, England (2002). 